\title{
DUALITY THEORY FOR GROTHENDIECK CATEGORIES ${ }^{1}$
}

\author{
BY ULRICH OBERST
}

Communicated by David Buchsbaum, May 5, 1969

In [3] Jan-Eric Roos has shown that an abelian category $\mathfrak{A}$ is a locally Noetherian Grothendieck category if and only if (iff) it is dual to the category of complete (and Hausdorff) topologically coherent left modules over some complete topologically left coherent and left coperfect ring $R$. In this note I announce a series of results which show similar relations for arbitrary, not necessarily locally Noetherian, Grothendieck categories.

Some preliminary notions are needed. A Grothendieck category is an abelian cocomplete category with exact filtered colimits and a family of generators. The dual of a Grothendieck category is a co-Grothendieck category. A topological ring $R$ is a ring (always unital) with a topology such that the addition and multiplication are continuous. A topological ring $R$ is called left linear topological $[1, \mathrm{p} .411]$ if 0 has a basis of neighborhoods consisting of left ideals. Such a basis of neighborhoods is simply called a basis of $R$. Given a topological ring $R$, a topological $R$-left module is an $R$-left module with a topology such that the addition on $M$ and the multiplication $R \times M \rightarrow M$ are continuous. A topological $R$-left module is called left linear topological if it admits a basis (of neighborhoods of 0 ) consisting of left submodules. Let $R$ be a left linear topological ring, and let $\operatorname{Dis}_{R} R$ be the category of discrete topological $R$-left modules. Then $D i_{R} R$ is a full subcategory of $\operatorname{Mod}_{R} R$, the category of all $R$-left modules, and consists of all $R$-left modules $M$ such that for all $m \in M$ the left annihilator $(0: m)$ is open in $R$. (Compare [3, $\S 4$, Proposition 3]). The category $\operatorname{Dis}_{R} R$ is the closed subcategory of $\operatorname{Mod}_{R} R$ associated with the given left linear topology on $R[1, \mathrm{p} .411 \mathrm{ff}]$. Let Coh $\left(\operatorname{Dis}_{R} R\right)$ denote the category of all coherent objects of $\operatorname{Dis}_{R} R[3, \S 5] ; \operatorname{Coh}\left(\operatorname{Dis}_{R} R\right)$ is a full, skeletal-small subcategory of $\operatorname{Mod}_{R} R$ and closed under finite limits and colimits (in $\operatorname{Mod}_{R} R$ ). In particular, $\operatorname{Coh}\left(\operatorname{Dis}_{R} R\right.$ ) is abelian. The ring $R$ is called topologically left coherent if $\operatorname{Dis}_{R} R$ admits a family of coherent generators, i.e. if $\operatorname{Coh}\left(\operatorname{Dis}_{R} R\right)$ is a class of generators for $\operatorname{Dis}_{R} R[3, \S 4$, Definition 3].

Let $R$ be any ring. An $R$-left module $M$ is called algebraically linearly compact if it satisfies the following condition: If $I$ is a directed ordered set and if $\left(M_{i} ; i \in I\right)$ is a decreasing family of finitely gene-

1 This research was supported by NSF grant GP-8425. 
rated submodules of $M$ then $\cap\left(M_{i} ; i \in I\right)$ is finitely generated and the canonical map $M \rightarrow$ proj $\lim M / M_{i}$ is surjective.

An $R$-left module $M$ is called coperfect $[3, \S 3$, Definition 2] if it satisfies the descending chain condition for finitely generated submodules. A coperfect $R$-left module is algebraically linearly compact. If $R$ is a right perfect ring then ${ }_{R} R$ is coperfect (see $[3, \S 3]$ for further references).

Let $R$ be any ring and $B$ a full subcategory of $\operatorname{Mod}_{R} R$, closed under finite limits and colimits. A module $B \in \mathfrak{B}$ is called $\mathfrak{B}$-linearly compact if it satisfies the following condition: If $I$ is a directed ordered set and if $\left(B_{i} ; i \in I\right)$ is a decreasing family of submodules $B_{i}$ of $B$ with $B_{i} \in \mathscr{B}$ then $\cap\left(B_{i} ; i \in I\right) \in \mathscr{B}$ and $B \rightarrow$ proj $\lim B / B_{i}$ is surjective. The latter limit is taken in $\operatorname{Mod}_{R} R$. I say that $\mathscr{B}$ is linearly compact if all its objects are $\mathbb{B}$-linearly compact. A ring $R$ is called algebraically left linearly compact if ${ }_{R} R$ is an algebraically linearly compact $R$ left module. For example, every right perfect ring is algebraically left linearly compact. A left linear topological ring $R$ is called topologically left linearly compact if it has a basis of open left ideals $a$ such that $R / \mathfrak{a}$ is an algebraically linearly compact $R$-module.

LEMMA 1. If $R$ is topologically left linearly compact then $\operatorname{Coh}\left(\operatorname{Dis}_{R} R\right)$ is linearly compact.

Again let $R$ be a ring and $\mathscr{B}$ a full, skeletal-small subcategory of $\operatorname{Mod}_{R} R$, closed under finite limits and colimits. Let $\hat{\mathfrak{B}}$ be the following category. An object $X$ of $\hat{B}$ is an $R$-left module together with a topology which makes $X$ into a complete (and Hausdorff) topological group and which admits a basis of $R$-submodules $X^{\prime}$ with $X / X^{\prime} \in \mathfrak{B}$. The continuous $R$-linear maps are the morphisms of $\hat{B}$. We consider $\mathfrak{B}$ as a full subcategory of $\hat{\mathfrak{B}}$ by equipping the modules in $\mathfrak{B}$ with the discrete topology. For $X \in \hat{\mathcal{B}}$, a submodule $X^{\prime}$ of $X$ is called special open if it is open and $X / X^{\prime} \in \mathfrak{B}$. A closed submodule $Y$ of $X \in \hat{B}$ is called special closed if $Y \in \hat{B}$ with respect to the induced topology. Finally an $X \in \hat{B}$ is called strict if any special closed $Y \subset X$ with $X / Y \in \mathscr{B}$ is open. Here $X / Y$ is just regarded as an $R$-module, no topology is considered on $X / Y$. Let $\tilde{B}$ denote the full subcategory of $\hat{\mathfrak{B}}$ of all strict modules.

Theorem 2. Assumptions as above. Assume that $\mathfrak{B}$ is linearly compact. Then $\mathfrak{B}$ is a co-Grothendieck category, and $\mathfrak{B}$ is a dense subcategory closed under taking subobjects, quotient objects, and finite coproducts in $\tilde{\mathfrak{B}}$.

That $\mathfrak{B}$ is a dense subcategory of $\tilde{\mathfrak{B}}$ means that each $X \in \tilde{\mathfrak{B}}$ is the 
limit of the projection functor $P_{X}: X \backslash \mathfrak{B} \rightarrow \mathfrak{B}$. Here $X \backslash \mathfrak{B}$ denotes the fiber of the injection functor $\mathfrak{B} \rightarrow \tilde{\mathfrak{B}}$ over $X$. The objects of $X \backslash \mathfrak{B}$ are pairs $(B, f)$ of an object $B \in \mathfrak{B} \subset \mathfrak{B}$ and a morphism $f: X \rightarrow B$ in $\tilde{\mathfrak{B}}$. One proves the preceding theorem by means of the following

Proposition 3. Assumptions as in the Theorem 2.

(i) The category $\hat{\mathfrak{B}}$ is complete and cocomplete. The kernel, cokernel resp. limit in $\hat{\mathfrak{B}}$ are those in $\operatorname{Mod}_{R} R$ with the induced, coinduced resp. limit topology. A morphism is a monomorphism resp. epimorphism iff it is injective resp. surjective.

(ii) The category $\mathfrak{B}$ is a reflexive subcategory of $\hat{\mathfrak{B}}$, i.e., the injection functor inj: $\mathfrak{\mathfrak { B }} \rightarrow \hat{\mathfrak{B}}$ has a right adjoint $s: \hat{\mathfrak{B}} \rightarrow \tilde{\mathfrak{B}}$ such that $s$ inj is isomorphic to the identity functor on $\mathfrak{\mathfrak { B }}$. In particular, if $\left(X_{i} ; i \in I\right)$ is an inverse system in $\tilde{\mathfrak{B}}$ then

$$
\text { proj } \lim _{\tilde{\mathfrak{B}}} X_{i} \cong s\left(\operatorname{proj} \lim _{\hat{\mathfrak{B}}} X_{i}\right) .
$$

Let now $R$ be a left linear topological ring. Let $T F\left({ }_{R} R\right)$ be the category of topologically finitely generated $R$-left modules. The objects of $T F\left({ }_{R} R\right)$ are the linear topological $R$-left modules $X$ which admit a basis of open submodules $X^{\prime}$ such that $X / X^{\prime}$ is finitely generated. The morphisms of $T F\left({ }_{R} R\right)$ are the continuous $R$-linear maps. Let $T C\left({ }_{R} R\right)$ be the full subcategory of $T F\left({ }_{R} R\right)$ of all topologically coherent $X$, i.e., those $X \in T F\left({ }_{R} R\right)$ which admit a basis of open submodules $X^{\prime}$ such that $X / X^{\prime} \in \operatorname{Coh}\left(\operatorname{Dis}_{R} R\right),[3, \S 5]$. Let $\operatorname{CTC}\left({ }_{R} R\right)$ be the full subcategory of $T C\left({ }_{R} R\right)$ of all complete (and Hausdorff) modules in $T C\left({ }_{R} R\right)$. Finally let $S T C\left({ }_{R} R\right)$ be the full subcategory of $C T C\left({ }_{R} R\right)$ of all strict modules. With $\mathfrak{B}:=\operatorname{Coh}\left(\operatorname{Dis}_{R} R\right)$ we have $\hat{\mathfrak{B}}=C T C\left({ }_{R} R\right)$ and $\tilde{B}=S T C\left({ }_{R} R\right)$. We obtain the full embeddings

$$
\operatorname{STC}\left({ }_{R} R\right) \subset \operatorname{CTC}\left({ }_{R} R\right) \subset T C\left({ }_{R} R\right) \subset T F\left({ }_{R} R\right) .
$$

Lemma 4. The category $C T C\left({ }_{R} R\right)$ is coreflexive in $T F\left({ }_{R} R\right)$, i.e., the injection functor

$$
\text { inj: } \operatorname{CTC}\left({ }_{R} R\right) \rightarrow T F\left({ }_{R} R\right)
$$

has a left adjoint

$$
\because \operatorname{TF}\left({ }_{R} R\right) \rightarrow C T C\left({ }_{R} R\right)
$$

such that ${ }^{\wedge}$ inj is isomorphic to the identity functor.

The functor ${ }^{\wedge}$ is called the coherent completion. In particular, ${ }_{R} R \in T F\left({ }_{R} R\right)$; hence $\hat{R} \in C T C\left({ }_{R} R\right)$. Also remark that the injection

$$
\operatorname{STC}\left({ }_{R} R\right) \subset \operatorname{CTC}\left({ }_{R} R\right)
$$


has the right adjoint $s$ (see Proposition 3). Hence $s(\hat{R}) \in S T C\left({ }_{R} R\right)$. A strict complete topologically left linearly compact and coherent ring is a left linear topological ring $R$ such that ${ }_{R} R \in S T C\left({ }_{R} R\right)$. Essentially as a corollary of Theorem 2 we obtain

THEOREM 5. Let $R$ be a topologically left linearly compact ring. Then the category $S T C\left({ }_{R} R\right)$ is a co-Grothendieck category with the projective generator $s(\hat{R})$. The category $\operatorname{Coh}\left(\operatorname{Dis}_{R} R\right)$ is a full, skeletal-small dense subcategory of $S T C\left({ }_{R} R\right)$, and closed under taking subobjects, quotient objects, and finite coproducts in $\operatorname{STC}\left({ }_{R} R\right)$. For each $X \in \mathrm{Coh}\left(\mathrm{Dis}_{R} R\right)$ there is a short exact sequence

$$
s(R)^{k} \rightarrow X \rightarrow 0, \quad k \text { a natural number },
$$

in $\operatorname{STC}\left({ }_{R} R\right)$.

The astonishing fact now is that indeed every co-Grothendieck category is equivalent to a category $\operatorname{STC}\left({ }_{R} R\right)$ as in Theorem 5 . Let $\mathfrak{A}$ be any Grothendieck category. As is easily seen there is a skeletalsmall, full subcategory $\mathfrak{N}$ of $\mathfrak{A}$ which is closed under taking subobjects, quotient objects and finite coproducts, and which is a class of generators for $\mathfrak{A}$. There also is an injective cogenerator $E$ of $\mathfrak{A}$ such that for each $N \in \mathfrak{N}$ there is a short exact sequence

$$
0 \rightarrow N \rightarrow E^{k}, \quad k \text { a natural number. }
$$

Let $\mathfrak{N}$ and $E$ be any such pair with the just described properties. If $A \in \mathfrak{A}$ then $\mathfrak{A}(A, E)$ is an $\mathfrak{A}(E, E)$-left module in the natural fashion. If $B \subset A$ identify $\mathfrak{A}(A / B, E)$ with its image under $\mathfrak{A}(A / B, E) \rightarrow \mathfrak{U}(A, E)$, i.e.,

$$
\mathfrak{A}(A / B, E)=\left\{f: A \rightarrow E ;\left.f\right|_{B}=0\right\} .
$$

Then there is a unique left linear topology on $\mathfrak{A}(E, E)$ which has the left ideals $\mathfrak{A}(E / N, E), N \subseteq E, N \in \mathfrak{N}$, as basis. Also, for $A \in \mathfrak{A}$, there is a unique left linear topology on the $\mathfrak{A}(E, E)$-left module $\mathfrak{H}(A, E)$ which has the submodules $\mathfrak{A}(A / N, E), N \subseteq A, N \in \mathfrak{N}$, as basis. These topologies are always meant when $\mathfrak{U}(A, E)$ is considered as a topological module.

TheOREM 6. Assumptions as above.

(i) The ring $\mathfrak{A}(E, E)$ is a strict complete topologically left linearly compact and topologically left coherent ring.

(ii) The assignment

$$
A \sim \mathcal{A}(A, E)
$$


defines the equivalence

$$
\mathfrak{\Re}^{\mathrm{op}} \rightarrow \operatorname{STC}(\mathfrak{A}(E, E)),
$$

and also the equivalence

$$
\mathfrak{N}^{\mathrm{op}} \rightarrow \operatorname{Coh}(\operatorname{Dis} \mathfrak{A}(E, E)) .
$$

The Theorems 5 and 6 show that an abelian category $\mathfrak{A}$ is a Grothendieck category iff it is dual to some category $\operatorname{STC}\left({ }_{R} R\right)$ where $R$ is a strict complete topologically left linearly compact and coherent ring. This is the announced generalization of the results of Jan-Eric Roos, indicated in the introduction.

COROLlaRy 7. Let $R$ be a strict complete topologically left linearly compact and coherent ring. Then there are a Grothendieck category $\mathfrak{A}$ and an injective cogenerator $E$ of $\mathfrak{A}$ such that $R \cong \mathfrak{Y}(E, E)$.

Examples. (1) $[3, \S 5]$ In the situation of Theorem 6 the following statements are equivalent:

(i) $\mathfrak{N}$ is Noetherian. (In this case $\mathfrak{N}$ is exactly the category of all Noetherian objects of $\mathfrak{A}$, and $\mathfrak{A}$ is locally Noetherian. For $E$ one can take the direct sum of a complete set of indecomposable injectives.)

(ii) $\mathfrak{A}(E, E)$ is topologically left coperfect.

(iii) $\operatorname{STC}(\mathfrak{A}(E, E))=C T C(\mathfrak{A}(E, E))$.

(2) Assume that $R$ is a left coherent and algebraically left linearly compact ring. With the discrete topology $\mathrm{R}$ is a strict complete topologically left coherent and linearly compact ring. The module ${ }_{R} R$ is a projective generator of $S T C\left({ }_{R} R\right)$. The category $\operatorname{Coh}\left(\operatorname{Mod}_{R} R\right)$ admits projective covers. Remark that in the situation of Theorem 6 the ring $R$ is discrete iff $E \in \Re$.

(3) Let $R$ be a right self-injective, regular ring, and let $\subseteq\left(R_{R}\right)$ be the full subcategory of $\operatorname{Mod} R_{R}$ of all direct summands of some product of copies of $R$. The category $\subseteq\left(R_{R}\right)$ is a spectral category, i.e., a Grothendieck category in which every morphism splits [2, Satz 2.2]. The module $R_{R}$ is a projective generator and an injective cogenerator of $\mathfrak{S}\left(R_{R}\right)$. Also, if $\mathfrak{A}$ is any spectral category with the generator $E$, then $\mathfrak{A}(E, E)$ is right self-injective, and regular, and the assignment $A \sim \mathfrak{A}(E, A)$ is an equivalence from $\mathfrak{A}$ to $\mathfrak{S}(\mathfrak{A}(E, E))$ [2, Satz 2.1]. Let $\mathfrak{N}$ be the full subcategory of $\mathfrak{S}\left(R_{R}\right)$ of all finitely generated projective $R$-right modules, i.e., of those $M \in \mathfrak{S}\left(R_{R}\right)$ which are direct summands of some $R^{k}, k$ a natural number. Then $E=R_{R}$ and $\mathfrak{N}$ satisfy the assumptions of Theorem 6 , and even $R_{R} \in \mathfrak{N}$. Moreover

$$
R \cong \mathscr{S}\left(R_{R}\right)\left(R_{R}, R_{R}\right)
$$


and the topology on $R$ is discrete.

THEOREM 8. Let $R$ be a right self-injective, regular ring.

(i) The ring $R$ is left coherent and algebraically left linearly compact.

(ii) The objects of the category $\operatorname{STC}\left({ }_{R} R\right)$ are exactly those of the form $s(Y)$ where $Y$ is a special closed submodule of some $R^{I}$, I any set. Here ${ }_{R} R$ has the discrete topology, and $R^{I}$ the product topology.

(iii) The assignment $M_{R} \leadsto \operatorname{Hom}\left(M_{R}, R_{R}\right)$ defines an equivalence

$$
\Im\left(R_{R}\right)^{\mathrm{op}} \rightarrow \operatorname{STC}\left({ }_{R} R\right) .
$$

Remark that the duality in (iii) generalizes the well-known duality between finitely generated projective $R$-right resp. $R$-left modules.

Another one of the many valuable results of Jan-Eric Roos in [3] states that the locally coherent Grothendieck categories are exactly those of the form $\operatorname{Lex}(\mathfrak{B}, A b)$ where $\mathfrak{B}$ is some small (or skeletalsmall) abelian category. The category $\operatorname{Lex}(\mathfrak{B}, \mathrm{Ab})$ is the category of all left exact, additive functors from $\mathfrak{B}$ to $\mathrm{Ab}$. $\mathrm{A}$ similar result holds in the more general situation of this note. Let $\mathfrak{B}$ be as in Theorem 2. Since $\mathfrak{B}$ is dense in $\tilde{\mathfrak{B}}$ we obtain the full embedding

$$
\begin{aligned}
& \tilde{\mathfrak{B}}^{\circ \mathfrak{p}} \longrightarrow \operatorname{Lex}(\mathfrak{B}, \mathrm{Ab}), \\
& \left.X \sim \tilde{B}(X,-)\right|_{\mathfrak{B} .}
\end{aligned}
$$

A morphism $f: G \rightarrow F$ in $\operatorname{Lex}(\mathfrak{B}, \mathrm{Ab})$ is called special if for every morphism $g: \mathfrak{B}(B,-) \rightarrow F, B \in \mathfrak{B}$, the fibered product (pull-back) $G \times{ }_{F} \mathfrak{B}(B,-)$ is representable. A subfunctor $G$ of $F$ in $\operatorname{Lex}(\mathfrak{B}, \mathrm{Ab})$ is called special if the injection $G \rightarrow F$ is special. A functor $F \in \operatorname{Lex}(\mathfrak{B}, \mathrm{Ab})$ is called strict if it satisfies the following two conditions:

(i) The functor $F$ commutes with intersections of subobjects. (Remark here that $\mathfrak{B}$ admits such intersections.)

(ii) If $G$ is a special subfunctor of $F$ and also a subfunctor of some representable functor then $G$ itself is representable.

Let $\operatorname{SLex}(\mathfrak{B}, \mathrm{Ab})$ be the full subcategory of $\operatorname{Lex}(\mathfrak{B}, \mathrm{AB})$ of all strict left exact functors.

Theorem 9. Assumptions as in Theorem 2. Then the functor $X \sim$ $\left.\tilde{\mathfrak{B}}(X,-)\right|_{\mathfrak{B}}$ defines an equivalence

$$
\tilde{\mathfrak{B}}^{\circ \mathrm{D}} \rightarrow \operatorname{SLex}(\mathfrak{B}, \mathrm{Ab}) \text {. }
$$

In particular, $\mathrm{SLex}(\mathfrak{B}, \mathrm{Ab})$ is a Grothendieck category. The category $\operatorname{SLex}(\mathfrak{B}, \mathrm{Ab})$ is locally Noetherian iff $\mathfrak{B}$ is Artinian. In this latter case $\operatorname{SLex}(\mathfrak{B}, A b)=\operatorname{Lex}(\mathfrak{B}, A b)$.

The preceding considerations imply 
THEOREM 10. Let $\mathfrak{A}$ be an abelian category. Then $\mathfrak{A}$ is a Grothendieck category iff it is equivalent to a category $\operatorname{SLex}\left(\operatorname{Coh}\left(\mathrm{Dis}_{R} R\right), \mathrm{Ab}\right)$ where $R$ is a strict complete topologically left linearly compact and coherent ring. The category $\mathrm{SLex}\left(\operatorname{Coh}\left(\operatorname{Dis}_{R} R\right), \mathrm{Ab}\right)$ is locally Noetherian iff $R$ is topologically left coperfect (see [3, §2]).

\section{REFERENCES}

1. P. Gabriel, Des catégories abéliennes, Bull. Soc. Math. France 90 (1962), 323448.

2. P. Gabriel and U. Oberst, Spectralkategorien und reguläre Ringe im von Neumannschen Sinn, Math. Z. 92 (1966), 389-395.

3. J.-E. Roos, Locally Notherian categories and generalized strictly linearly compact rings. Applications, Proceedings of the Batelle Conference on Categorical and Homological Algebra, Lecture notes, vol. 92, Springer-Verlag, New York, 1969.

University of Chicago, Chicago, Illinois 60637 